\title{
América Latina frente al desafío de las tecnologías de la información y la comunicación
}

\author{
Enrique Olivares RodrígueZ*
}

\begin{abstract}
The current state in informatics, derived from technological change and the global capitalist expansionism, has affected dramatically the international markets as well as the technological paths that sustain industrial development. This is also true for the scientific basis activities. At the same time, the enterprise restructuring that has transformed the Fordist-Taylorist productive process, from the Keynesian standpoint on flexible production, has had a profound impact in all aspects of the system: with the incorporation of the manufacturing systems in the context of informatics and mechatronics, as well as information and communication technologies (ICT). This restructuring has defined the relationship between industrial innovation and academia in all countries, above all in those that set the production pace and dominate the international market.
\end{abstract}

Keywords: ICT, globalization, technological change, flexible production, Latin America.

\section{Resumen}

Las condiciones de la informática derivadas del cambio tecnológico y del expansionismo capitalista mundial han afectado sensiblemente los referentes del mercado internacional y de las trayectorias tecnológicas en que se sustenta el desarrollo industrial, sobre todo a aquellas actividades de base científica. Asimismo, la reestructuración empresarial que ha motivado la transformación del proceso productivo fordista-taylorista desde la directriz keynesiana en producción flexible ha repercutido profundamente en todos los ámbitos del sistema a partir de la incorporación de los sistemas manufactureros en el contexto de la informática y la mecatrónica, y en general las tecnologías de información y comunicación (TIC). Tales reestructuraciones han redefinido la relación entre las condiciones de la innovación industrial y la académica en todos los países, principalmente en aquellos que marcan el ritmo de desenvolvimiento de la producción y su dominio sobre el mercado internacional.

Palabras clave: TIC, mundialización, cambio tecnológico, producción flexible, América Latina.

\footnotetext{
*Universidad Autónoma Metropolitana, Unidad Xochimilco.

Correo-e: jeoliva@correo.xoc.uam.mx.
} 


\section{Evolución de las tecnologías de la información y la comunicación (TIC), y desarrollo humano ${ }^{1}$ \\ 1.1. ¿Del siglo de las luces al siglo de las penumbras tecnologizadas?}

Aquella fase que observa una transformación del capitalismo monopolista de base nacional, estadocéntrico y predominantemente industrial-financiero (1900 a 1970) al capitalismo, monopolizado u oligopolizado, cada vez más desnacionalizado, 'desterritorializado', trasnacionalizado, parasitario y especulativo, así como fundamentalmente sustentado en los sectores terciarios (servicios, finanzas, información, cultura) y cuaternarios (científico-técnicos) de la economía (Rafael Cervantes et al., "La metamorfosis del capitalismo monopolista", Cuba Socialista, en Suárez, 2000), o en lo que se conoce mejor como globalización, descansa esencialmente y está siendo impulsada por las TIC (incluidos los adelantos en el transporte y el empleo del ciberespacio) en el marco de la revolución científico-tecnológica.

Sus más recientes descubrimientos y el empleo (aún no generalizado) de nuevas fuentes de energía (atómica, solar, eólica, mareomotriz, la celda de hidrógeno, la biomasa, etc.) se complican con la pretensión -en el ámbito aeroespacial- de dominar monopólicamente el cosmos y, aún más inmediatamente, el desarrollo técnico militar, como por ejemplo la denominada "guerra de las galaxias", la guerra del golfo, "la guerra contra el mal”, el terrorismo, y así por el estilo; y también la biotecnología (con sus posibilidades de clonar y modificar las especies y sus aplicaciones científico-tecnológicas de la ingeniería informacional), la producción de nuevos materiales sintéticos y nuevas aleaciones fisicoquímicas, la informática y la cibernética, lo cual no sería factible sin el surgimiento de nuevas disciplinas y el fraccionamiento de otras, como la física del estado sólido, la cristalografía o la ingeniería genética. Lo anterior sin dejar de reconocer los desiguales efectos de la informática y la cibernética sobre la existencia humana, y en particular en lo que René Dreyfuss (1997) denominó "la reorganización tecnotrónica" de la producción, de los servicios y otras dimensiones simbólico-culturales del mundo; así como la emergencia de nuevas y diversas actividades ocupacionales y su caudal de síndromes (en cuanto a sus formas de enajenación,

${ }^{1}$ La presente colaboración se presentó como ponencia en el $51^{\circ}$ Seminario Internacional de Americanistas, "Repensando las Américas en los umbrales del siglo xxI", del 14 al 18 de julio de 2003, en Santiago de Chile. 
fetichización y devaluación de valores -sobre todo humanos-) sobre la población.

En el presente se advierten severos y complejos cambios, en términos crecientemente adversos a la sociedad; el discurso se encuentra preñado de amenazas (y aun cumplimientos de ellas) atentatorias contra el inconformismo, y de oposición ante, verbigracia, los referidos a crímenes de lesa humanidad. El poder omnímodo y su avance implacable sin contrapeso ni atenuación crecen ante las victorias pírricas y aun ante la reprobación mundial. Ni las sanciones morales ni la descalificación de los altos comisionados y de las instituciones "reconocidas internacionalmente" son diques frente a la prepotencia y soberbia que encarnan las ambiciones supranacionales coaligadas para imponer su voluntad e intereses. La tecnología y la ciencia de la destrucción (aquí orientadas y diseñadas para amedrentar a la oposición irrestrictamente) han dejado su papel neutral para adoptar el carácter agresivo-disuasivo frente a potenciales o reales barreras a los designios imperialistas. Imperialismo apoyado o "justificado" por ataques aún no localizados ni suficientemente esclarecidos, pero cuyo revanchismo y "castigo ejemplar" no dejan lugar a dudas, pese a que los destinatarios o "cabezas de turco" (árabes o cubanos, o cualquiera que sea no blanco o no oligarca) resulten ser finalmente no culpables, ni con mucho comprobados sus supuestos crímenes. Si bien a la postre los sacrificados resultan ser -en su mayoría- víctimas inocentes de entre civiles (niños, jóvenes, mujeres y ancianos) que no incurrieron en mayor falta que vivir en territorios o sistemas no gratos al Primer Mundo.

Las armas de destrucción masiva usadas en Irak constituyen el perfeccionamiento de la alta tecnología bélica (en gran medida vinculada con las TIC) empleada en décadas previas en Hiroshima y Nagasaki, y más recientemente en Irán, Bosnia e Irak, entre otros, y resultan en "laboratorios" que incluyen el desarrollo espacial, la "guerra de las galaxias", los transbordadores del espacio exterior, y así por el estilo, cuyo fin es perfeccionar el control y la hegemonía no lograda mediante el uso doméstico de la tecnología. Armas, y en particular los misiles 'inteligentes', de profundidad, de fraccionamiento, de penetración nocturna, diseñadas sobre todo para 'ablandar' a distancia, cuyos daños 'colaterales' (torres de telecomunicación -y sus correspondientes equipos de TV y transmisión satelital-, hospitales que incluyen la Cruz Roja, hoteles y construcciones de uso civil) no tienen nada de accidental ni de inesperados. Los saldos que arroja la 
mutilación, y los daños infligidos especialmente a la población civil, dan cuenta del éxito alcanzado por la destrucción "creativa" del moderno imperialismo, en sus pretensiones de supremacía total y globalizada.

Las alianzas establecidas para "derrotar" a los enemigos del imperialismo norteamericano (supuestos o reales) son reveladoras de los intereses creados en torno a las empresas trasnacionales (ETN), creadas a la sombra de la Segunda Guerra Mundial y del reparto triádrico del mundo. Estados Unidos junto con el Reino Unido (y Japón desde luego), se ha 'asociado' a partir de alianzas estratégicas, capital de riesgo y otras figuras administrativas, con base en las TIC (aunque no necesariamente sólo con ellas), las parcelas de la componenda mercantil. No es fortuito que frente a tales alianzas, el eje francoalemán (entre otros motivos, petroleros y mercantiles, desde luego) se haya opuesto a convalidar los motivos guerreristas (expansivos e invasores, así sea por intereses particulares), por la supremacía triádrica, en los nuevos mercados internacionales.

\subsection{Tendencias recientes de las TIC en el mundo}

De cara al mercado mundial, las actuales las tendencias observadas son:

- El capitalismo monopolista ha "industrializado" la superestructura (la cultura y los medios de comunicación) tanto por su función económica directa (producción y venta de nuevas mercancías) como por la indirecta: la publicidad y otros servicios culturales y la instauración de su dominio político e ideológico (mediante el control de "los medios de producción mental”) (Suárez, 2000).

- La creciente tendencia a la baja de los costos de las Tic, y en particular de los gigabyts, en el mercado informacional ha expandido aceleradamente la penetración de los megaoligopolios de teleinfocomunicaciones.

- El ciberespacio es controlado por unas cuantas megacorporaciones, con base en las TIC, de TV (alta resolución), de fibra óptica (terrestre y/o ultramarina, de banda ancha, de comunicación satelital, celular, entre otras), cuyos ejes combinan el control de la base de datos, la telecomunicación audiovisual y a distancia (la TV de alta resolución); y la comunicación por cable ha sustituido los viejos conductores de cobre y aluminio por la fibra óptica, cuya capacidad de 
conducción y manejo de datos ha permitido el transporte audiovisual (de sonido e imágenes y datos) en banda ancha.

- Cien de ellas tienen la hegemonía en la comunicación visual, a distancia y 'neuronal' de las TIC, y tan sólo 10 de ellas controlan el sector de las telecomunicaciones y la microelectrónica, y en su mayoría son de origen estadounidense.

- Cinco o seis megaempresas o empresas mundiales lanzarán cientos de satélites de baja órbita para rastrear los "sistemas de comunicación móviles globales” (Suárez, 2000), lo que profundizará el control, operación y construcción de nuevas redes locales y regionales, así como mundiales, junto con la perseguida búsqueda de compatibilidad entre los diversos sistemas existentes; asimismo, los nuevos servicios de videotelex, el teletex, la transmisión celular inalámbrica, la comunicación digital y los bancos de datos online o virtuales. En todo ello ha sido determinante el control en la investigación y desarrollo (I-D) y la investigación y desarrollo experimental (IDE) mediante superredes de información y colaboración de las ETN y aun pequeñas y medianas empresas (pymes) muy especializadas a partir de alianzas estratégicas para la investigación y el desarrollo de procesos y productos conjuntamente entre las grandes, medianas y pequeñas empresas de alta tecnología. La colaboración industrial en I-D establecida entre Estados Unidos y el resto del mundo puede apreciarse en sus alcances para el año 2002, y sus mayores repercusiones se vislumbran en la distribución a partir de los montos asignados por las ETN y sus afiliadas (veáse gráfica $\mathrm{I}^{2}$ ). Son de destacarse los acuerdos de inversión establecidos entre Estados Unidos y sus filiales, sobre todo con los países nórdicos ( $\$ 15.8$ billones de dólares corrientes), con la Unión Europea (\$10.6), con Japón (\$3.2), con Canadá (\$2.4), y así por el estilo, con Medio Oriente y el cono Sudamericano.

La reducción de los costos de pista vocal, de audio y de video, así como el incremento en la capacidad de conducción de las mismas, han sido determinantes en el expansionismo de las TIC y en el control que sobre ellas ejercen las ETN. Los precios de las computadoras declinan, parejamente con la inversión en I-D e IDE

${ }^{2}$ Las gráficas y los cuadros de referencia se encuentran en el anexo del presente trabajo. 
de los monopolios informáticos (véanse gráficas II a IV). En la gráfica III en particular podemos apreciar los fuertes desembolsos de los países miembros de la Organización para la Cooperación y el Desarrollo Económico (OCDE) y los partícipes del Grupo de los Siete (G-7) como los más representativos de tales incrementos, seguidos por Estados Unidos y Japón, y, al final, los montos erogados por los países no miembros de la OCDE y no miembros del G-7. En la gráfica $\mathrm{V}$ se puede ver la alta prospectiva de crecimiento hasta el año 2010 de la telefonía móvil, en contraste con la declinación de la fija.

Los antecedentes de todo se encuentran, desde luego, en la curva logarítmica que sirvió como base a Gordon Moore para la formulación de la ley que lleva su nombre (gráfica vi) y en los porcentajes (ya en particular desprendidos de la gráfica III, de los países más industrializados) en I-D como porcentaje del producto interno bruto respectivos, donde encabeza la lista Japón, seguido por Estados Unidos, Alemania y Francia. Destaca la grave caída de Rusia, que logra recuperarse y ponerse a la par de Italia; en tanto que México, en contraste, no llega a alcanzar en ningún momento ni el uno por ciento (véase gráfica VII).

\section{Nueva división del trabajo y papel de las empresas mundiales $^{3}$}

En reestructuración productiva, y a partir de la distribución triádrica del mercado, son de subrayar los siguientes resultados:

- La reestructuración productiva de la posguerra, la crisis posbélica y los nuevos requerimientos del sistema imponen un nuevo régimen de producción ante la caída de la tasa de ganancia y el desarrollo en los mercados nacionales e internacionales. Las necesidades de ahorro (de medios de producción, de fuerza de trabajo e insumos) viabilizan el paso del fordismo a la producción flexible, la cual se logra mediante la introducción de alta tecnología (AT) en los procesos.

- Los nuevos sistemas productivos se integran a partir de la informática y la comunicación instantánea.

${ }^{3}$ Para este apartado se consultaron las obras de Luis Suárez (2000) y Alonso Aguilar (2002), fundamentalmente. 
- Las ETN han establecido una asignación de tareas a partir de la ubicuidad productiva (que en casos extremos llega a la desindustrialización), a la maquila y el ensamblado aun 'en tránsito' hacia los 'puntos de venta'.

- La desregulación ha acelerado la expansión exponencial de las teleinfocomunicaciones, pero su aplicación fue sólo confirmatoria (de la lógica del capitalismo) de los nuevos procesos. La 'destrucción creativa', como lo confirman las experiencias recientes del combate a "las fuerzas del mal" (en Irak), convalida el expansionismo trasnacional. Otros factores se encuentran presentes sobre todo en la acelerada penetración de la telefonía celular, la falta de infraestructura en líneas fijas, el alto nivel oferente de las empresas operadoras, y el valor de signo (el estatus social), así como las amplias facilidades y oportunidades que ofrecen muchos gobiernos, como paraísos fiscales a las compañías operadoras (del sudeste asiático, de América Latina y de otros países sobre todo subdesarrollados; véase gráfica IX).

Si se compara el ritmo de difusión de los aparatos más representativos de la Sociedad de la Información, podrá vislumbrarse la capacidad del cambio tecnológico y la innovación a que han llegado las Tic. Mientras que al teléfono le llevó 74 años alcanzar los 50 millones de usuarios, en tanto que la radio tuvo que esperar 38 años para alcanzar la misma cifra, a las computadoras personales sólo les llevó 16 años, 13 a la TV, y tan sólo cuatro años a la red de internet (véase gráfica VIII). Sus operaciones, convalidando las tendencias de la producción en general, resul$\tan$ en que:

- El comercio se realiza sobre todo intrafirmas (más que interfirmas), lo que abate grandemente los costos y fracciona la producción (las teleinfocomunicaciones y las redes de bancos de datos de I-D, y de diseño, CAD/CAM/Cim [diseño asistido por computadora, manufactura asistida por computadora, e integración manufacturera asistida por computadora], agilizan los procesos).

- La ciencia y la tecnología, la I-D y la IDE captan en gran medida los recursos empresariales, sobre todo las de base científica. 
3. Nueva división internacional del trabajo e internacionalización de la producción

\subsection{Internacionalización, desregulación y "libre comercio"}

Uno de los principales nuevos rasgos de la internacionalización es que la producción misma se internacionaliza y aun se globaliza. Las grandes empresas, aunque en algunos casos intervenían en cierta medida en actividades productivas, en un momento dado se interesaron no sólo en comprar y vender en esos mercados, o incluso en tener una pequeña participación de la producción, sino que empezaron a producir ellas mismas en escala cada vez mayor. A partir de entonces, la producción se fragmenta, y lo que antes se hacía en un lugar determinado y con bastante unidad, ahora procedería de diversas instalaciones y aun de diferentes países, a veces muy alejados entre sí. Factores de diferente naturaleza influyeron en esa internacionalización. Y desde la segunda mitad de los años sesenta, la baja de la tasa de beneficio, o el temor de que ésta declinara donde la producción había ya aumentado sustancialmente, contribuyó sin duda a que la producción se desplazara hacia el exterior en ciertas actividades. Tanto la revolución de los transportes y las comunicaciones como las nuevas tecnologías, y con frecuencia, sobre todo, las nuevas formas de organización, permitieron reducir costos, diversificar la producción y mejorar su calidad. Pero, ante la necesidad de dar respuesta con rapidez a nuevas y crecientes demandas de productos diferenciados, que conforme a las exigencias de la moda y la presión de una severa competencia eran a menudo pronto sustituidos por otros, la organización fordista resultaba demasiado rígida, pesada, costosa, incapaz de responder adecuadamente a las nuevas exigencias de los compradores e incluso inadecuada para introducir con éxito nuevas tecnologías (CAD, CAM y СIM), así como un control de calidad, el incremento de productividad, el desarrollo de proveedores; pero, sobre todo, el nuevo más intenso aprovechamiento de la mano de obra y en particular de los conocimientos implícitos que dejaba sin explotar en su provecho la producción fordista-taylorista; así como el diseño flexible y altamente versátil y justo a tiempo, como lo requerían los nuevos tiempos. Se introdujeron cambios sustanciales: no para el mercado en general sino para la demanda solvente.

La internacionalización de la producción no alteró de fondo la estructura económica de los países industrializados y los subdesarrollados; trajo consigo una nueva división internacional del 
trabajo. La hegemonía de Estados Unidos se debilita frente al rápido ascenso de Alemania y Japón, así como frente a la industrialización de los tigres del sudeste asiático. Aun países como Brasil y México absorbieron fuertes inversiones extranjeras que les permitieron producir ciertos bienes durables, así como maquinaria y equipo que antes importaban en su totalidad. Las ETN, que operaban a veces bajo regímenes como las "franjas de libre exportación" o maquiladoras, aun totalmente extranjeras, fueron importantes y modificaron la economía y el comercio exterior de los países receptores, sobre todo cuando se asociaron con capitales domésticos. Lo que impide realmente a nuestros países un desarrollo relativo comparable, por ejemplo, con el de los nuevos países industrializados, es la intensificación de la dependencia estructural (económica, científica, tecnológica e ideológica), sobre todo de Norteamérica, y la debilidad del régimen estatal, de sometimiento de las sucesivas administraciones del gobierno mexicano.

Las empresas dominantes en la internacionalización de la producción han sido casi siempre poderosos consorcios trasnacionales, que operan como oligopolios, en forma de redes, ahora más estrechamente ligados entre sí y a menudo aliados con grandes empresas de otros países, todo lo cual se expresa en altos grados de concentración y, a la vez, en menor integración vertical y en cierta descentralización operativa, que a menudo resultan más que de la creación de nuevas empresas, de la fusión y adquisición de negocios ya establecidos.

La verdadera revolución de la tecnología en las comunicaciones y los transportes, y especialmente el espectacular avance de las tecnologías de la información, han hecho posible que la economía y otras actividades se internacionalicen como nunca antes.

Entre las ETN, las pyme y otras de diverso nivel y alcance se han establecido vínculos apoyados directa o indirectamente por el Estado; y en general la infraestructura de ciencia y tecnología, entre otras a partir de alianzas estratégicas, capital de riesgo y otros mecanismos, que a menudo se establecen por razones tecnológicas, ha contribuido a que la internacionalización alcance creciente impulso, y en particular a que la investigación y el desarrollo sean mayores, y también a que se internacionalicen cada vez más. Desde una posición tecnologista podría pensarse que la tecnología es la lámpara maravillosa. En la realidad, en la internacionalización de la tecnología, el intercambio de información a partir de las redes, el intercambio interempresarial y aun entre los centros de 
investigación (públicos y privados) existen intereses y compromisos coincidentes y decisivos, sin los cuales no se comprendería lo que acontece. Uno fundamental es la ciencia, sin cuyos avances algunas nuevas tecnologías serían imposibles, o al menos se desarrollarían muy lentamente; y otro es la educación y capacitación, es decir, el adiestramiento de quienes han de operarla.

Una buena selección de técnicas es muy importante en una estrategia de desarrollo. Y el que ciertas tecnologías destruyan más empleos que los que crean es en gran parte algo que corresponde resolver a la sociedad y a la organización económica en su conjunto.

Porque mientras estén presentes las desigualdades y los desajustes que caracterizan a la economía del subdesarrollo, la mejor política es aquella que al mismo tiempo que incorpora modernas tecnologías, mediante cuantiosas inversiones que eleven el nivel de productividad, amplía las posibilidades de trabajo y ocupación de quienes quizá por mucho tiempo no tendrán acceso a esas tecnologías, pues aun así pueden contribuir a elevar el nivel de producción y de ingreso, así como a mejorar sus condiciones de vida (Aguilar, 2002).

Resultado de ello es la nueva estructuración productiva mundial (sobre todo en productos de AT) (Suárez, 2000).

\subsection{Distribución asimétrica de las TIC}

3.2.1. Tendencias recientes del mundo de internet (World Wide Web en el 2001)

Un servidor web es un tipo especial de servicio soportado por un ordenador central que proporciona información en el popular formato World Wide Web utilizado por navegadores (browsers) como Explorer o Netscape. El número de servidores web ha aumentado espectacularmente: pasó de 75,000 a finales de 1995 a más de 25 millones a finales de 2000 . El crecimiento tan sólo en ese año fue de 158\%, el más elevado desde 1998. Los servidores web representan ahora $25 \%$ de todos los ordenadores centrales de internet. Los servidores que terminan con el "codiciado sufijo" .com son 15 millones y representan 57\% del total. El sufijo de dominio de país con más servidores web es .uk, del Reino Unido, con 1.7 millones; es decir, 7\% del total. Los sitios web más populares son los de Yahoo!, el portal internet que fue visitado por unos 90 millones de usuarios en noviembre de 2000. 
En los albores de este nuevo milenio, casi todos los países están conectados a internet. Ello, sin embargo, no debe suponer que existe una conexión en todos los estratos y en todos los sectores sociales. En 1988 sólo siete países estaban conectados a la red de la National Science Foundation de Estados Unidos, un decenio más tarde ya eran 200 y en 1997 casi todos tenían una conexión a internet. Actualmente, menos de media docena de economías no están conectadas, principalmente por motivos políticos. La ventaja de internet es que, desde la primera conexión, y por muy lenta que sea, ofrece todo lo que tiene. También es importante ser el primero: de los siete primeros países que se conectaron a internet de Estados Unidos, es decir, Canadá, Dinamarca, Finlandia, Francia, Islandia, Noruega y Suecia, todos, salvo Francia, están entre los 10 países con mayor penetración de internet.

En el entorno mundial, la internet ha sido considerada como una pequeña parte del fenómeno globalizador. La red ha sido incrementada de 213 host de computadora y varios miles de usuarios en agosto de 1981, a más de 56 millones de host de internet hacia julio de 1999 (gráfica XI), soportando un estimado de 190 millones de usuarios de internet. Sin embargo, aún más imprevisible es el número de ciudades conectadas a la red global. Al cerrar el siglo Xx en julio de 1999, cerca de 200 países estaban conectados. Las tendencias mostradas en la gráfica véase gráfica VII eran imprevisibles. Un estudio detenido de las cifras de la gráfica XI revela las grandes disparidades de los host de internet entre regiones de alto y bajo ingreso. Por ejemplo, encontramos casi los mismos host en Francia que en toda América Latina y el Caribe. Hay más host en tres países altamente desarrollados de la región Asia-Pacífico (Australia, Japón y Nueva Zelanda) que en otras naciones de la región combinadas, y hay más host en Nueva York que toda África. La mayoría de los host internet están en los países desarrollados, lo que sugiere que una buena salud y una óptima educación son los factores definitorios la difusión de la internet.

El perfil de los usuarios de internet confirma que éstos tienen, en promedio, alto estrato social y nivel educación, y que asimismo son jóvenes, urbanos, líderes en su campo y de sexo masculino. En contraste, nos preguntaríamos, ¿cuáles son las barreras para incrementar el uso de internet? En realidad existen diferentes niveles de obstáculos, y se encuentran de acuerdo con el nivel del desarrollo económico y social de cada país, pero 
los usuarios alrededor del mundo coinciden unánimemente en ubicar el mayor obstáculo en el precio del acceso a internet como su mejor promotor. Los precios del acceso a internet para los usuarios finales pueden considerarse a la baja por tres componentes: el hardware/software, la amplia disponibilidad del acceso a internet y los cargos por el servicio telefónico. En términos relativos, los costos para conectarse son mucho más altos en los países subdesarrollados. En lo anterior, los precios pueden no ser drásticamente diferentes en términos absolutos. En los hechos existe un desfase muy amplio entre los países de alto y de bajo ingreso cuando se consideran los costos relativos de ingreso per cápita.

\subsubsection{Distribución de las TIC en el mundo}

- En los países desarrollados existen 312 computadoras centrales para 10,000 personas en promedio.

- En los países subdesarrollados existen seis computadoras centrales para 10,000 personas en promedio.

- Canadá y Estados Unidos absorben 61\% de los Tic; la Unión Europea, 24.3\%; Australia, Japón y Nueva Zelanda, 7\%; en tanto que otros países absorben 4.6\%.

- Los países subdesarrollados del sudeste asiático absorben 2.8\% de las TIC; América Latina y el Caribe, 1.2\%, y África, 0.5\% (véase la gráfica X) (UIT, 2001).

La significativa escasez de infraestructura en las líneas telefónicas es un nuevo gran obstáculo para incrementar el acceso a la internet en los países subdesarrollados. La notable fortaleza de internet y la conciencia de su crecimiento e importancia en las TIC para el desarrollo socioeconómico están cambiando las guías de las políticas para la construcción de redes que incrementen los servicios telefónicos. Los países están profundizando en el estudio de este problema con una amplia variedad de opciones, incluyendo el involucramiento comprometido de las compañías operadoras, a las que se les permite más libertad para la reinversión de sus ingresos y una fuerte contracción de las inversiones del sector privado mediante inversiones compartidas en las compañías telefónicas estatales, y/o por el desarrollo de nuevos mercados emergentes (véase gráfica XI). (UIT,1999a). Lo anterior, aunque en gran parte es aplicable, casuísticamente, plantea cuestiones muy discutibles, como el hecho de que las TIC en la experiencia 
de las economías subdesarrolladas, y con mayor razón en las pobres como África y el Caribe, entre otras, no han mejorado sustancialmente los niveles de vida generales, ni mucho menos reducido los porcentajes de miseria o, como se suele denominar eufemísticamente a tales sectores, la "pobreza extrema" de tales economías.

En el año 2000 empezaron a utilizar la internet unos 80 millones de usuarios, y el total en la alborada del siglo XXI es de unos 315 millones. Aproximadamente 5\% del mundo está en línea (esto significa que $95 \%$ del resto del mundo no está conectado a internet). Aunque la tasa de crecimiento en los mercados subdesarrollados es de casi el doble que en los países desarrollados y ahora representan aquéllos casi una cuarta parte de todos los usuarios de internet, la brecha digital sigue siendo muy grande. Si bien casi la tercera parte de los habitantes de los países desarrollados están en linea, son menos de $2 \%$ en los países subdesarrollados. No es sorprendente que el lugar de nacimiento de la internet, Estados Unidos, sea el mayor mercado del mundo, con casi 100 millones de usuarios a principios de 2001. El segundo es Japón, con unos 39 millones de usuarios, de los cuales más de la mitad pueden acceder también a internet desde teléfonos móviles. China ha experimentado un espectacular crecimiento de internet para un país en desarrollo y es ahora el tercer mercado, con 23 millones de usuarios. La minúscula Islandia es el lugar más conectado del mundo, ya que $60 \%$ de su población es usuaria de la internet.

La demanda de mayor ancho de banda se atendió inicialmente con líneas de la red digital de servicios integrados (RDSI, sus siglas en inglés), que funciona con líneas telefónicas tradicionales. La RDSI de tarifa básica ofrece dos líneas y ha tenido mucho éxito entre los usuarios que no desean bloquear su línea telefónica cuando navegan por la red, aunque sólo ofrece una velocidad marginalmente superior (64 kbit/s) que el acceso de marcación directa convencional. A principios del 2000 había unos 24 millones de abonados RDSI en el mundo, principalmente en Norteamérica, Europa Occidental y Japón, que representaban 7\% de todas las líneas telefónicas en servicio en esa época. En esa fecha, menos de 3\% de los abonados a la internet podían disponer de un acceso local de banda ancha tal como la línea de abonado digital asíncrona (ADSL) o el módem de cable (UIT, 2001).

El año 2000 representó un paso histórico para la capacidad de la internet internacional, ya que por primera vez rebasó la capaci- 
dad de los circuitos telefónicos internacionales. La capacidad de la internet internacional en todo el mundo era de casi $300 \mathrm{Gbit} / \mathrm{s}$; es decir, casi cinco veces más que en 1999. El precio de la internet es un factor importante del volumen de acceso, y se compone de dos elementos: el primero es el precio facturado por el ISP (proveedores de servicio internet), que puede ser un canon fijo para una utilización ilimitada o por cierto número de horas, o un precio por tiempo. El segundo elemento del precio es la tasa de utilización del servicio telefónico local. En algunos países no se facturan las comunicaciones locales, o se facturan por un precio fijo. Países como Canadá, que no factura las telecomunicaciones locales, tienen las tarifas más bajas y altos niveles de penetración de la internet. En el otro extremo, países como Bélgica, con un acceso a internet supuestamente 'gratuito'; es decir, que el ISP no presenta una factura separada, pueden tener unas tarifas muy elevadas si las llamadas locales también lo son (véanse gráficas X y XI).

De lo anterior se concluye que existe una severa asimetría en la distribución de las TIC (que con fines ilustrativos hemos sintetizado mediante las simbolizadas por el ancho de banda de la internet internacional). En la cúspide de uso se encuentran Estados Unidos y Canadá, conectados con Europa (en 56 Gbit/s), y con Asia-Pacífico (en $18 \mathrm{Gbit} / \mathrm{s})$. En contraste, sólo se dispone de $3 \mathrm{Gbit} / \mathrm{s}$ para comunicarse con América Latina y de $0.5 \mathrm{Gbit} / \mathrm{s}$ para comunicarse con África, lo que permite apreciar las altos claroscuros existentes entre los usuarios (emisores/receptores) de tales tecnologías (véase gráfica XI). Destacan las siguientes estadísticas:

- El 96\% de las computadoras centrales de internet (CCI), se encuentra en los países de alto ingreso, cuya población constituye tan sólo $16 \%$ de la población mundial.

- En el hecho anterior destaca que únicamente Finlandia cuenta con más CCI que el conjunto de los países agrupados en América Latina y el Caribe.

- Y que en la ciudad de Nueva York, por ejemplo, se encuentran instaladas más CCI que en toda el África.

\subsection{Megacorporaciones triádricas que integran los consorcios de ordenadores}

El reparto del mercado en los tres grandes bloques mercantiles articulados a partir de la posguerra mundial: Estados Unidos, la Unión Europea y el sudeste asiático, con Japón a la cabeza, se ha 
establecido de manera similar a las corporaciones monopólicas previas, salvo que las actuales alcanzan características y dimensiones mundializadas, y arracimadas en nuevos espacios territoriales. Los consorcios de ordenadores, en sendos espacios triádicos, son liderados por Estados Unidos, y luego por Japón y la Unión Europea, los cuales han establecido intrincadas y espesas redes de vinculación, tanto en la producción como en la distribución de los procesos y productos de AT. Y, desde luego, mediante la intercomunicación y el intercambio de I-D e IDE, de experiencias y resultados de sus laboratorios nacionales y de conocimientos y avances en nuevos productos, materiales, procesos y servicios.

Las tendencias a la concentración y a la centralización de las megaempresas se encuentran guiadas por "la gigante" IBM, la General Automation y la Hewlett-Packard; en Japón, por la Nippon Electronic Corporation, la Matsushita y, compartiendo el siguiente sitio, la Takeda Riken y la Mitsubishi Jimuki; en tanto que para la Unión Europea, la Bull (de Francia), la Secomsa (del gobierno español), y la Siemens (de Alemania), por sólo nombrar los primeros tres lugares ocupados en tales grandes mercados (para una referencia más detallada véase la tabla 1 anexa, y Ohmae, 1991).

Por lo que corresponde a la telefonía fija y móvil, tienen una interrelación directa con la conexión a las redes de la internet mundiales (como lo fue desde sus inicios en Estados Unidos con el Pentágono, con el proyecto Arpanet), lo que permite establecer parámetros para vislumbrar la predominancia e instrumentación ejercida por los países industrializados sobre las TIC, y en especial las estrechas ligas de las ETN y el desarrollo de la industria bélica. No es gratuito que los principales productores de armamento, de manera directa o indirecta, se encuentren involucrados en las actividades militares ocurridas en las décadas de fines del siglo xx e inicios del XxI. Así, encontramos que los principales exportadores de armamentos en el mundo son: Estados Unidos, Rusia, Francia, Reino Unido y Alemania (Bilan du Monde, París, 1998, citado por Suárez, 2000).

\section{El neomonroísmo y las asimetrías Norte-Sur}

De los puntos previos se desprenden las siguientes secuelas:

- Las crisis, la desregulación y el neoliberalismo han originado la recolonización norteamericana sobre los países subdesarrollados, en especial en América Latina. 
- Un camino para la recolonización económica de la región han sido los acuerdos: de inversión y libre comercio (Acuerdo Multilateral de Inversiones, Área de Libre Comercio de las Américas, Tratado de Libre Comercio, Organización Mundial de Comercio [OMC] y OCDE).

- Estados Unidos pretende estructurar las "autopistas electrónicas de la información en las Américas”.

- En la Segunda Cumbre de Santiago de Chile se propuso establecer un comercio electrónico libre de impuestos en todo el hemisferio (a partir de los acuerdos con México, Costa Rica, Argentina y Brasil); ello ha favorecido la liberalización y privatización de todos los servicios de telecomunicaciones.

- Lo anterior ha derivado en las décadas perdidas de América Latina y de gran parte de los países subdesarrollados (Suárez, 2000: 210).

- El acceso a las TIC se vuelve crecientemente inalcanzable para los países subdesarrollados.

- En tanto que en las economías africanas y del Medio Oriente se convierte en inaccesible, lo que abre más la brecha.

- En las economías africanas no se habla de dimensionar la penetración celular, sino del tiempo empleado en el tránsito de los usuarios para tener acceso a algún teléfono (fijo o móvil), que va de 15 a 60 minutos de caminar a pie (véase cuadro 1).

- El comercio internacional ha crecido más que la producción.

- Tales empresas mundiales son las que producen, venden, emplean e invierten más capital fuera de sus países de origen que en ellos, y que al paso del tiempo son crecientemente poderosas. En cierto momento, tales empresas incentivaron a muchos países subdesarrollados a endeudarse como nunca antes; después les impusieron políticas de desregulación y reestructuración que los obligaban a gastar e importar menos y a exportar más para pagar sus cuantiosas deudas, atrayéndolos con la posibilidad de entrar al mercado de Estados Unidos, lo que en realidad pocos países han logrado. Y, en algunos casos, aprovecharon estos países para abrir en ellos franjas de "libre exportación" o maquiladoras, que les permitieron relocalizar ciertas actividades o fases del proceso productivo a fin de reducir costos, sobre todo salariales, y tener una mejor ubicación geográfica para surtir atractivos mercados. 
Y dos hechos de diferente naturaleza, que en cierto modo se refuerzan mutuamente, explican el porqué de esa desregulación. El primero es la prolongada crisis que siguió a la fuerte caída de la actividad económica de mitad de los años setenta, crisis que hizo ver no sólo la menor eficacia de ciertos mecanismos reguladores, sino también la imposibilidad, o al menos la inconveniencia, de tratar de utilizarlos, e incluso el deterioro de todo el sistema o modo previo de regulación. Y el segundo es la política neoliberal que unos años más tarde empezó a aplicarse, y que, como se sabe, es antiestatista y antirregulacionista; es decir, considera que el mercado libre, dejado a su suerte, cumple mejor su función y asignará racionalmente los recursos, lo que no ocurrirá si se interfiere con él, por medio de un tipo u otro de medidas reguladoras (en alguna medida coincidiendo o convalidando las posiciones neoclásicas del equilibrio de mercado, aunque aquellas llegan a reconocer la 'endogeneidad' de los factores tecnológicos).

La alta velocidad y volumen de los mercados financieros y su relación con la internacionalización de sus mercados se deben a los medios de comunicación (en buena medida las transacciones son realmente "electrónicas). También son importantes la mayor actividad financiera -ya no meramente industrial o comercial- de las grandes corporaciones, las cuantiosas operaciones que hoy se realizan con acciones de empresas extranjeras, y la expansión de las bolsas y mercados de valores, y sobre todo de los mercados de cambios, cuyos precios y activos tienen una alta volatilidad financiera.

Incluso en algunos países subdesarrollados, las posibilidades que hoy se abren para su desarrollo son mayores que las de épocas previas, lo que en parte obedece a que la creciente integración subordinada a los países más fuertes implica una mayor presencia del capital extranjero trasnacional en esos países, que obliga a otorgar facilidades y aun brindar apoyos que antes se negaban, y a que algunas de las viejas y más restrictivas formas de dominación imperialista se superen. Y, a la vez, los países más poderosos, con Estados Unidos en la punta, arbitrariamente intervienen en los asuntos internos de otras naciones, violan su soberanía, hacen la guerra (así llamado eufemísticamente lo que en realidad representa una invasión expansionista implacable) y se autoerigen en jueces a los que supuestamente corresponde decidir lo que deba hacerse. Al respecto se da actualmente una situación muy contradictoria, pues por un lado la dependencia es incluso mayor que antes, sobre todo en los países subdesarrollados, donde 
especialmente la política económica no es formulada por ellos sino por las grandes potencias de las que dependen en mayor medida y por los organismos financieros internacionales más influyentes (Banco Mundial y Fondo Monetario Internacional); y, por el otro, los países que aceptan esa subordinación y cuentan con recursos y con un nivel de desarrollo que les permite aumentar su capacidad productiva, tienen hoy cierto acceso a tecnologías, mercados y recursos financieros de los que anteriormente carecían, y que les posibilitan reestructurar sus economías, aunque a la vez han tenido que pagar un alto precio que se expresa, entre otras formas, en la dramática desigualdad social y en el aumento sin precedente de amplios sectores de la sociedad que viven en la miseria (Aguilar, 2002).

El alcance de la desregulación, la privatización y el desmantelamiento mismo del sector público es un asunto que el Estado decide, aunque bajo la presión del capital nacional y extranjero. En el contexto de la mundialización, y sobre todo de la internacionalización del capital, el mundo se mueve en forma casi exclusiva bajo el capitalismo, y en consecuencia económicamente más atrasados son los países subdesarrollados, ya no precapitalistas. Tal es el caso de América Central, así como de otros países latinoamericanos. Incluso casi todos los países latinoamericanos -Ecuador, Perú, Bolivia-, así como Malasia y Tailandia, en Asia, o Argelia, Túnez o Marruecos, en África.

\section{Conclusiones}

Los principales cambios se advierten, particularmente, en que algunos afectan profundamente las relaciones de producción, y en que entre los países persiste y aun se ha agravado una dominación-dependencia que autoriza a pensar que el imperialismo no ha sido superado; la dominación que hoy ejercen los países del Grupo de los Siete, con Estados Unidos al frente, interviene ilegalmente en los asuntos internos de otros países no sólo en los procesos internos, sino aun de manera insolente y ostensible en los que debieran ser los organismos reguladores e institucionalizadores del acontecer mundial, como la Organización de las Naciones Unidas y sus aláteres, así como los financieros internacionales y la OMC. La reciente agresión bélica a Irak y la amenaza permanente a los países discrepantes, así como otras agresiones e imposiciones menos visibles pero no por ello menos dañinas, también suelen imponer, sobre todo a endeudadas y débiles nacio- 
nes subdesarrolladas, condiciones que limitan su libertad, estorban y desvían su desarrollo y lesionan su soberanía. Y pese a que algunas grandes potencias no comparten las políticas belicistas o tienen contradicciones en ocasiones no tan secundarias, de alguna manera también siguen beneficiándose de su posición dominante en la relación con otros países, y en particular con los subdesarrollados. En el trato entre ellas -que sin duda sigue siendo el más importante-aun no han desaparecido la competencia, los desacuerdos, la rivalidad y ciertos motivos de conflicto. Tanto entre Estados y gobiernos como entre empresas trasnacionales de diferentes países se advierten actualmente formas de negociación y aun de cooperación que no estaban presentes o eran muy débiles hace años, y que hoy son la base de acuerdos transfronterizos y celebración de alianzas estratégicas, sobre todo cuando hay intereses empresariales y de coincidencia en la profundización de la I-D, la IDE y sobre todo de las TIC, para seguir manteniendo sus respectivas hegemonías y distribución triádica de los mercados.

Puesto que el Estado decide, presionado por los capitales nacional y extranjero, el alcance de la desregulación, la privatización y el desmantelamiento del sector público, la capacidad de acción estatal es una variable que depende del tipo de Estado, de su organización y de la amplitud y carácter de las fuerzas en que descansa. Los Estados son parte del proceso de globalización; pero a medida que las economías nacionales se internacionalizan, la autonomía relativa del Estado tiende a reducirse. La propia burguesía nacional, en países donde antes dirigió el proceso de acumulación de capital, se debilita frente a los países más poderosos y los organismos financieros internacionales, que ahora intervienen directamente y aun deciden el tipo de política que se adopta.

El cambio que la mundialización entraña, acaso sea un cambio de mayor dimensión. A partir de la idea de que el gran capital monopolista $-y$ quizá cada vez más oligopolista- sigue siendo con mucho el dominante, podría decirse que persiste el imperialismo. Pero tendría que aceptarse que éste no es el viejo imperialismo estudiado por los clásicos, sino un nuevo imperialismo que desborda y modifica profundamente al de hace un siglo, y cuya dinámica interna habría que comprender a fondo; esto es, el lugar que ocupa en la historia, la dirección en que se orienta y su relación con el capitalismo y con posibles nuevas formas de organización de la sociedad. 
Hoy en día podría decirse que hay varios niveles claramente diferenciados en los que el capitalismo se desenvuelve. Por ejemplo, el primero es el de las economías más desarrolladas, entre las que también hay diferencias, pero en conjunto se caracterizan por tener un alto grado de desarrollo, operar con tecnologías avanzadas y cuantiosas inversiones nacionales y extranjeras, y contar con amplios mercados (Aguilar, 2002).

Un segundo nivel corresponde a países que han tenido un rápido desarrollo económico en los últimos tres o cuatro decenios, donde podría situarse a los llamados 'nuevos países industrializados' del sureste de Asia.

Una siguiente modalidad muy peculiar del capitalismo se encuentra en lo que fueron la Unión Soviética y los países de Europa del Este. Al parecer, se trata de un capitalismo parasitario, que depende todavía en gran medida de lo que fue la economía estatal-socialista previa, en el que ha habido pocas inversiones nuevas realmente productivas, y en el que la privatización ha sido un mecanismo que auspicia la corrupción y los negocios fáciles y sucios.

Otro nivel podría ser el de países como Brasil y México, que pese a seguir siendo economías subdesarrolladas y dependientes han logrado significativos cambios, modernizando y diversificando su estructura económica, avanzando en el proceso de organización e industrialización, y ampliando sus mercados internos y, a veces, sobre todo externos; pero que al mismo tiempo tienen todavía sectores de la economía muy atrasados, economías con bajo nivel de integración, una profunda desigualdad social y una alta proporción de la fuerza de trabajo subempleada y que vive en condiciones de "extrema pobreza". Y, en contrapartida, ello resulta en un polarizado nivel de concentración; esto es, en contrastantes niveles de ingreso, en los cuales las capas medias continúan proletarizándose.

Y el último nivel podría corresponder a numerosos países sobre todo de Asia y África- en los que si bien el capitalismo se ha vuelto también el modo de producción dominante, continúan siendo atrasados y pobres. Han quedado en cierto modo al margen de la mundialización del capital y siguen dependiendo en buena medida de la producción y exportación de materias primas (Aguilar, 2002). 
Siglas

AT: Alta tecnología.

CAD: Diseño asistido por computadora.

CAM: Manufactura asistida por computadora.

CCI: Computadores centrales de internet.

CIM: Integración manufacturera asistida por computadora.

ETN: Empresas trasnacionales.

I-D: Investigación y desarrollo.

IDE: Investigación y desarrollo experimental.

ISP: Proveedores de servicios de internet.

OMC: Organización Mundial de Comercio.

OCDE: Organización para la Cooperación y el Desarrollo Económico.

Pyme: Pequeñas y medianas empresas.

RDSI: Red digital de servicios integrados.

TIC: Tecnologías de la información y la comunicación

Anexo

\section{Gráfica I}

Gasto en investigación y desarrollo de industrias en Estados Unidos y afiliadas en el extranjero por región del mundo, 1998 (miles de millones de dólares corrientes)

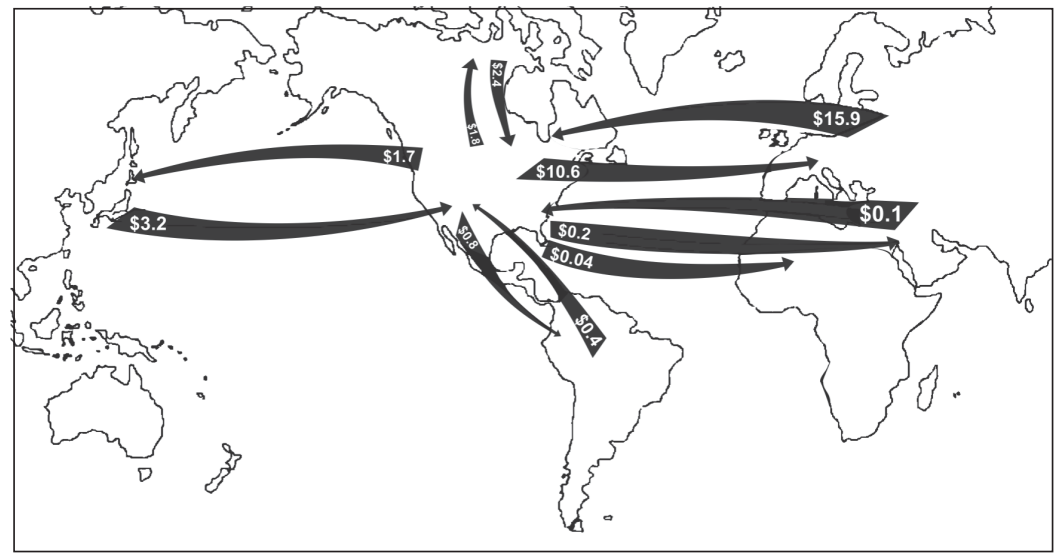

Fuente: Elaborada con base en datos de Science \& Engineering Indicators (2002). 


\section{Gráfica II}

Infraestructura de la capacidad y del costo. Cables trasatlánticos (1985-2000)

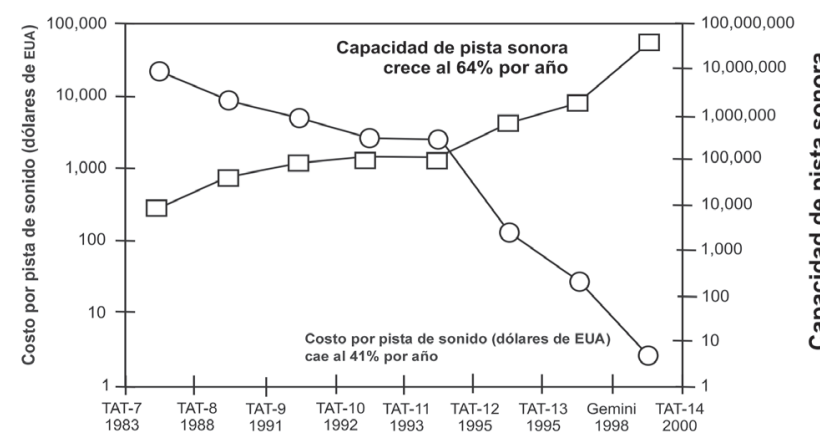

TAT: Capacidad y costo de cables trasatlánticos.

Fuente: Elaborada con datos de UIT (1999a).

\section{Gráfica III}

Gastos en investigación y desarrollo en Estados Unidos y países de la Organización para la Cooperación y el Desarrollo Económico

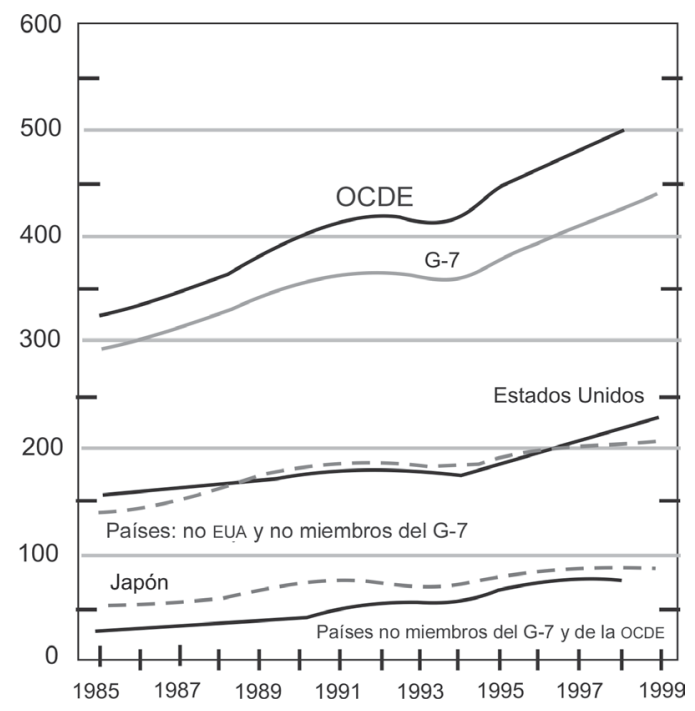

Fuente: Elaborada con datos de la OCDE (2001). 
Gráfica IV

Caída de precio de las computadoras.

Escala de precios: $1996=100$

Escala de precios: $1996=100$

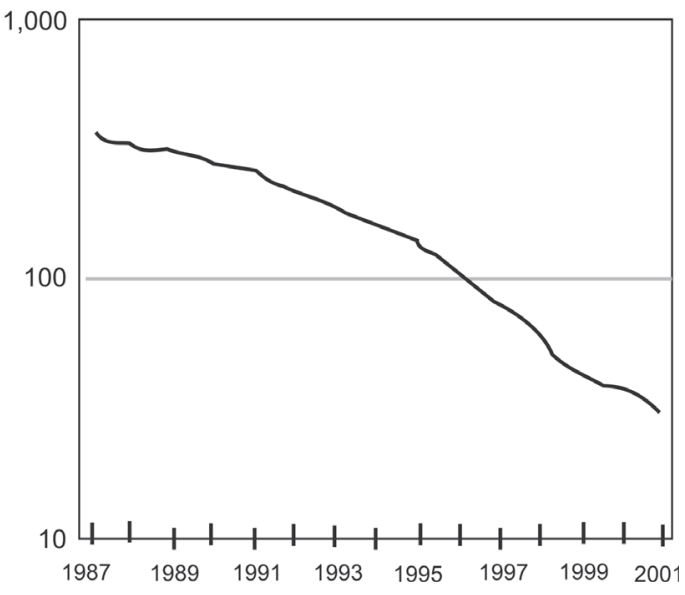

Fuente: Elaborada con base en datos de u.s Department of Commerce, Bureau of Economic Analysis, National Accounts Data, en Science \& Engineering Indicators (2002: c.8-5).

\section{Gráfica v}

\section{Millones de abonados al servicio telefónico en todo el mundo}

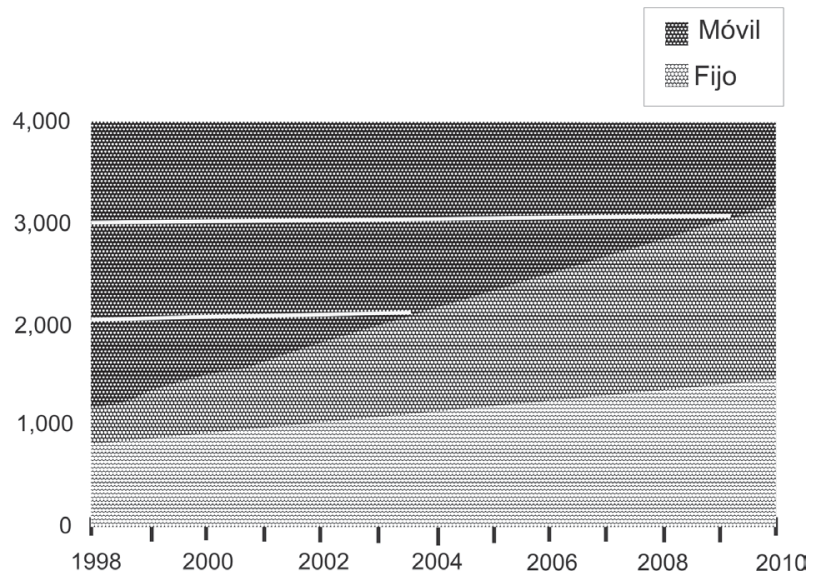

Fuente: Elaborada con base en datos sobre indicadores mundiales de las telecomunicaciones de la UIT, 1990-1998, y proyecciones de la UIT para después de 1998 (UIT, 1999b). 


\section{Gráfica VI}

\section{Ley de Moore, 1971-2005}

Número de transistores (escala de registro)

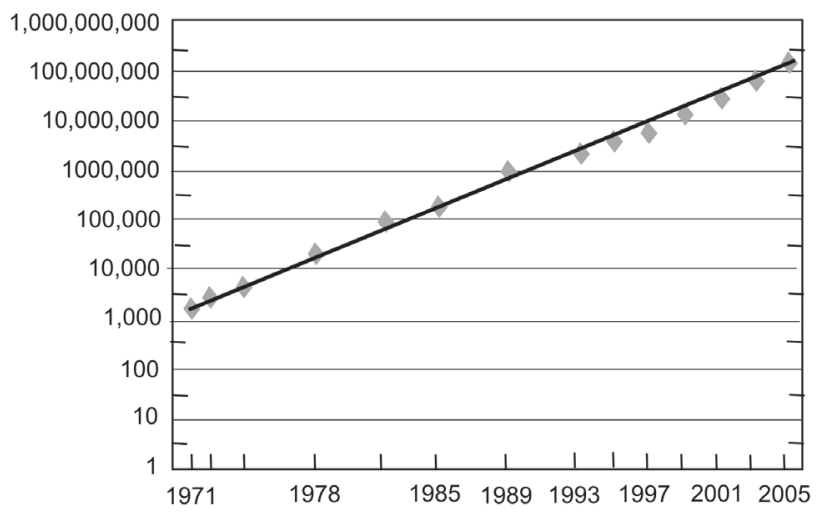

La línea en la gráfica representa la tendencia definida por la Ley de Moore. Los puntos representan (entre 1971-2001) los datos reales y la proyección de los datos (entre 2003-2005).

Fuente: Elaborada con datos de Science \& Engineering Indicators (2002).

\section{Gráfica VII \\ Investigación y desarrollo como porcentaje del PIB en los países del G-8}

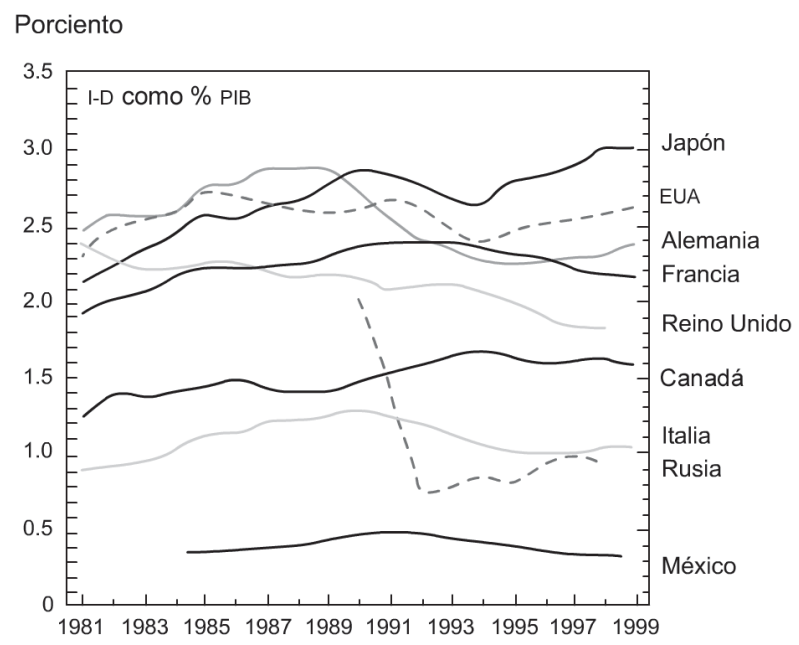

Fuente: Elaborada con base en datos de Science, Technology and Industrial Scoreboard (2003). 


\section{Gráfica VIII}

Ritmo de difusión de las tecnologías de la información y la comunicación (años que han sido necesarios para llegar a 50 millones de usuarios)

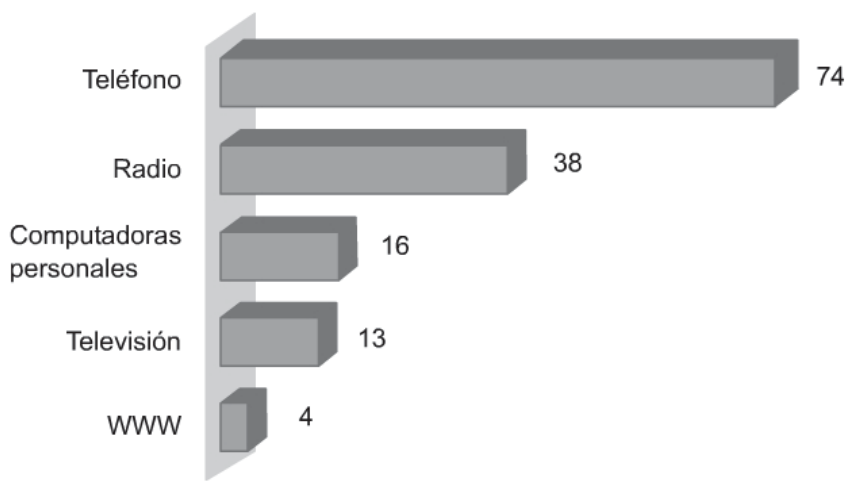

Fuente: UIT (1999a).

\section{Gráfica IX}

\section{Crecimiento mundial de las tecnologías de la información y la comunicación}

Ingresos e ingresos previstos en el mundo, en miles de millones de US\$

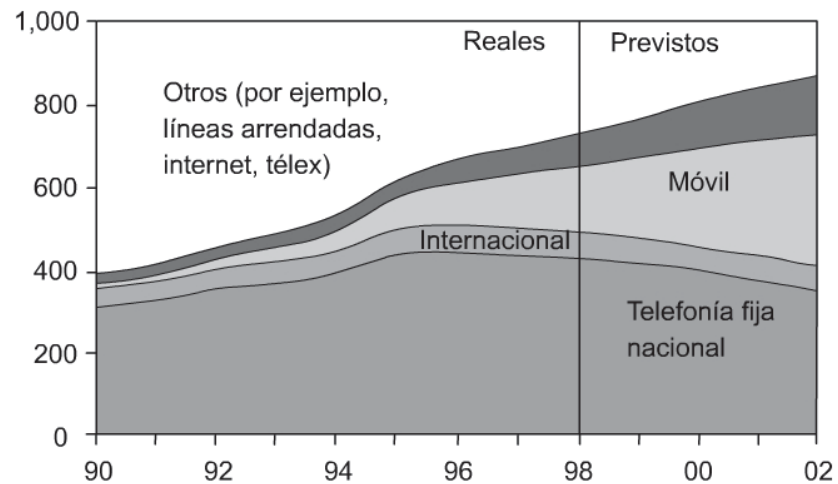

Fuente: Elaborada con base en datos sobre indicadores mundiales de las telecomunicaciones de la UIT, 1990-1998, y proyecciones de la UIT para después de 1998 (UIT, 1999b). 


\section{Gráfica $\mathrm{x}$ \\ Distribución de las tecnologías de la información y la comunicación en el mundo}

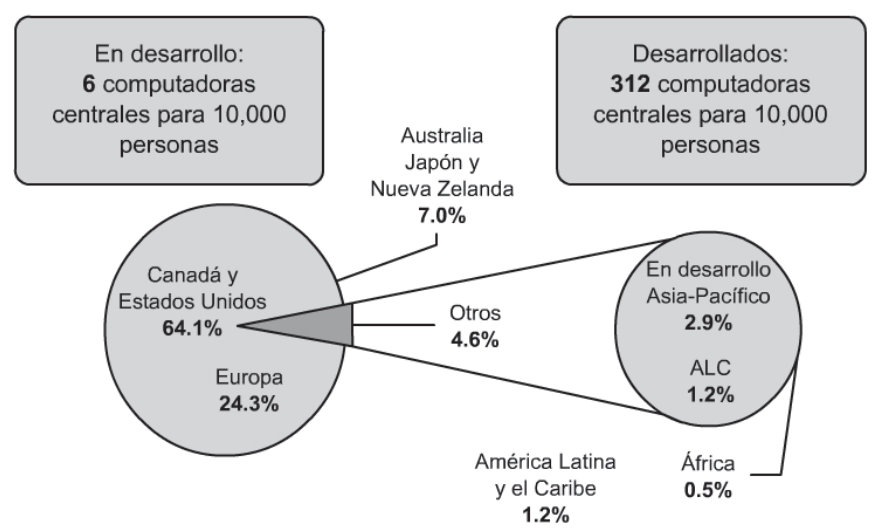

Fuente: Elaborada con base en UIT (2001).

\section{Gráfica XI}

Crecimiento desigual de los host de internet en el mundo

Internet hosts, worldwide, millones

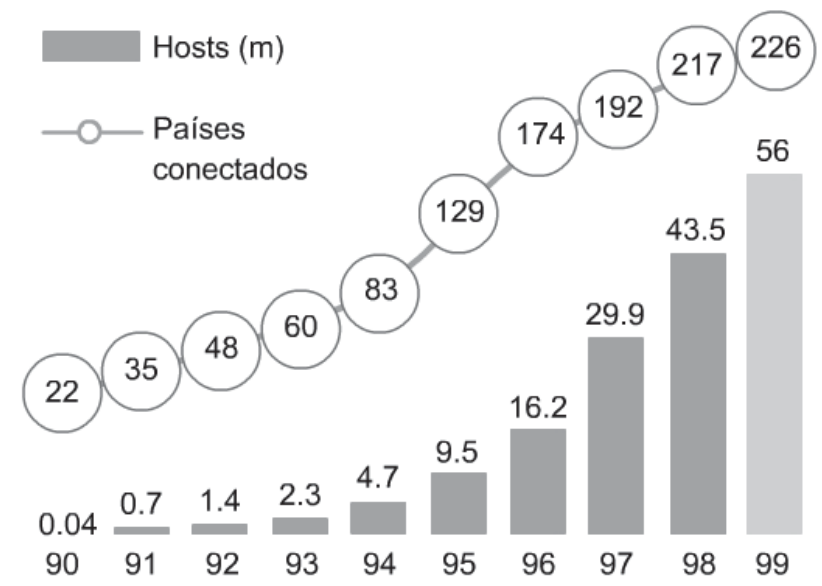

Fuente: UIT (1999a). 


\section{Cuadro 1 \\ Sudáfrica: acceso a teléfono \\ (porcentajes)}

\begin{tabular}{lccc}
\hline & Todos & De color & Blancos \\
\hline Servicio universal & 34 & 20 & 89 \\
$\begin{array}{l}\text { Acceso universal } \\
(15 \text { min. caminando) }\end{array}$ & 71 & 60 & 99 \\
$\begin{array}{l}\text { Acceso universal } \\
(30 \text { min. caminando) }\end{array}$ & 83 & 78 & 100 \\
$\begin{array}{l}\text { Acceso universal } \\
(60 \text { min. caminando })\end{array}$ & 90 & 87 & 100 \\
\hline
\end{tabular}

Fuente: Elaborado con base en Goldstein y O'Connor (2002: 178).

\section{Tabla 1}

Megacorporaciones triádicas: consorcios de ordenadores

\begin{tabular}{|c|c|c|}
\hline EUA & Japón & $\begin{array}{l}\text { Unión } \\
\text { Europea }\end{array}$ \\
\hline Honeywell 80\% & Nippon Lect Co. & Bull (Francia) \\
\hline IBM & Matsushita & Gob. español \\
\hline Gral. Automation 50\% & Takeda Riken 20\% & Siemens (Alem.) \\
\hline Hewlett-Packard 49\% & Mitsubishi Jimuki 20\% & ICL Ltd (GB) (75\%) \\
\hline Amdahi 50\% & Toshiba & Basf (Alem.) (75\%) \\
\hline TRW 49\% & Kayo Electron & Olivetti (Italia) \\
\hline National Cash Register & Yokogawa Electron & Nixdori/Alemania \\
\hline Nasco $34.7 \%$ & Fujitsu & $\begin{array}{l}\text { GmbH (Deutchland) } \\
\text { (Alem.) }\end{array}$ \\
\hline Sperry Univac 45\% & Mitsu \& Co. & \\
\hline Data General 85\% & Mitsubishi Electric & \\
\hline \multirow[t]{5}{*}{ Wang Computer 100\% } & Oki Electric & \\
\hline & Kosokelkaku Eng. & \\
\hline & C Itah Data $100 \%$ & \\
\hline & Kanematsu Gosha & \\
\hline & Casio & \\
\hline
\end{tabular}

Fuente: Elaborada con base en Ohmae (1991) y Suárez (2000). 


\section{Bibliografía}

Aguilar M., Alonso (2002), Globalización y capitalismo, Plaza y Janés, México.

Dreyfuss, René (1997), A época das perplexidades, $2^{\mathrm{a}}$ ed., Voces, Petrópolis.

Goldstein, Andrea y David O'Connor (eds.) (2002), Electronic Commerce for Development. International Development, Development Centre, OECD, París.

OECD (Organization for Economic Co-operation and Development) (2000), Science, Technology and Industrial Scoreboard, OECD, París. (2001), Main Economic Indicators, OECD, París.

Ohmae, Kenichi (1991), El poder de la tríada: las nuevas reglas de la competencia mundial, McGraw Hill, México.

Science \& Engineering Indicators (2002), National Science Foundation, Arlington, va.

Suárez Salazar, Luis (2000), El siglo XXI, posibilidades y desafíos para la Revolución cubana, Ciencias Sociales, La Habana.

UIT (Unión Internacional de Telecomunicaciones) (1999a), Challenges to the Network. Internet for Development, Executive Summary, UIT, Ginebra.

(1999b) Informe sobre el desarrollo mundial de las telecomunicaciones. Telefonía celular. Resumen analítico, UIT, http://www.itu.int/ITU-D/publications/wtdr_99/material/ wtdr99s-es.pdf.

(2001), Actualización de los indicadores de telecomunicaciones, UIT, Ginebra.

Recibido: 3 de mayo de 2004. Reenviado: 24 de agosto de 2005. 
Reenviado: 27 de septiembre de 2005. Reenviado: 23 de mayo de 2006. Aceptado: 5 de junio de 2006.

Enrique Olivares Rodríguez lleva ya 24 años como profesor-investigador de la Universidad Autónoma Metropolitana, unidad Xochimilco. Cuenta con posgrados en economía (Facultad de Economía en la UNAM) y en diseño (División de CyAD en la UAM). Destacan entre sus publicaciones los ensayos: "Reforma educativa para la enajenación obrera", en Reforma educativa y apertura democrática (1972); "Gobierno, corrupción y sindicalismo", en La burguesía mexicana (1973); "Política burguesa en el movimiento obrero", en Control y luchas del movimiento obrero (1978); "El desarrollo de la tecnología en la industria nacional", en Tecnología, universidad y autonomía nacional (1991); "Modernización industrial o la estrechez de la trayectoria tecnológica", en Cambio tecnológico y modernización industrial en México (1995), además de otros libros científicos, revistas, periódicos y memorias. Es también autor de Economía y tecnología en la industrialización de México, UAM-X, México, 1990; México: crisis y dependencia tecnológica, UAM-X-ENT, México, 1993, y México: mundialización y competitividad industrial en el cambio tecnológico (en prensa). 